\title{
Monitoring Of Toxic Gases and Measuring the Trace Amounts Of Metals
}

\author{
Sauvik Das Gupta ${ }^{1}$, Souvik Ghosal ${ }^{2}$, Rajesh Bag ${ }^{3}$, Subhajit Ghosh ${ }^{4}$, \\ SK Masud Hossain ${ }^{5}$, Abhishek Mallik ${ }^{6}$ \\ ${ }^{1,6}$ School of Electrical and Computer Engineering, Oklahoma State University, Stillwater, OK, USA \\ 2,3,4,5 West Bengal University of Technology, Kolkata, West Bengal, India
}

\begin{abstract}
The part of the spectrum that is particularly useful in identifying and measuring pollutants consists of radiation that interacts with the atoms and molecules that make up life on Earth. This includes radiation in the visible, and IR (infrared) regions. Many pollutants can be identified by their IR spectra. Because all molecules that absorb strongly at specific wavelengths exhibit spectral fingerprints. Wavelengths emitted constitute a unique "fingerprint" for each element and their intensity reflects the metal concentration. Spectral fingerprint is obtained from the wavelengths of light that each element absorbs.

This system utilizes an Infrared gas sensor for specific response of target gas. A sensor is used to monitor a specific range of the IR spectrum corresponding to the signature wavelength of the target gas. When the target gas passes between an IR source and the detectors, the absorption spectrum changes and instrument electronics are used to process this information and determine gas concentration. This system subsequently can provide audible and visual alarm indications. Toxic gases such as hydrogen sulfide, ammonia, styrene, hydrogen fluoride can be monitored using this technique. Trace amounts of certain metals such as mercury and arsenic are more accurately measured from their absorption.
\end{abstract}

Keywords: IR Spectrum : Spectral Fingerprints : IR Gas Sensor

\section{Introduction}

With our eyes we see the world in visible light. Whereas visible light falls only a small part of the radiation spectrum, the invisible light covers most of the remaining spectral range. The radiation of invisible light carries much more additional information. Each body with a temperature above the absolute zero($273.15^{\circ} \mathrm{C}=0$ Kelvin) emits an electromagnetic radiation from its surface, which is proportional to its intrinsic temperature.

With the help of a lens (input optics) the beams are focused on a detector element, which generates an electrical signal proportional to the radiation. The signal is amplified and, using successive digital signal processing is transformed into an output signal proportional to the concentration of gases.

A spectrum in the physical sense is the intensity of a mixture of electromagnetic waves as the function of the wavelength or frequency. The electromagnetic radiation spectrum covers a wavelength area of about 23 decimal powers and varies from sector to sector in origin, creation and application of the radiation. All types of electromagnetic radiation follow similar principles of diffraction, refraction, reflection and polarization. Their expansion speed corresponds to the light speed under normal conditions. The result of multiplying wavelength with frequency is constant: $\mathrm{f}=\mathrm{c}$.

The infrared radiation covers a very limited part in the whole range of the electromagnetic spectrum: It starts at the visible range of about $0.78 \mu \mathrm{m}$..Atoms of different elements may be thought of as having different arrangements of electrons around the nucleus in increasing energy levels. When metals such as lead, copper, and cadmium are vaporized at high temperatures, some electrons jump to higher energy levels. When the electrons drop to their original levels, the metal atoms emit radiation in a range of wavelengths from IR to UV, including visible light. The colors in fireworks result from such emissions. The wavelengths emitted constitute a unique "fingerprint" for each element and their intensity reflects the metal concentration 


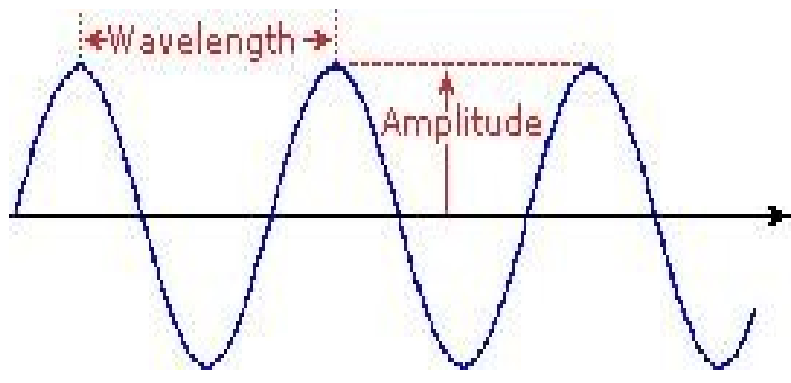

Figure 1. Wavelength and amplitude of a function

The same spectral fingerprint is obtained from the wavelengths of light that each element absorbs. Trace amounts of certain metals such as mercury and arsenic are more accurately measured from their absorption, rather than their emission spectra.

Many pollutants can be identified by their UV and IR spectra because all molecules that absorb strongly at specific wavelengths exhibit spectral fingerprints. Pollutants are often detected by spectroscopy. Gases such as those from vehicle emissions, landfills, industrial manufacturing plants, electric power plants, and hazardous incineration smokestacks can be monitored by spectroscopic methods. Gas and chemical leaks may also be monitored by spectroscopy.
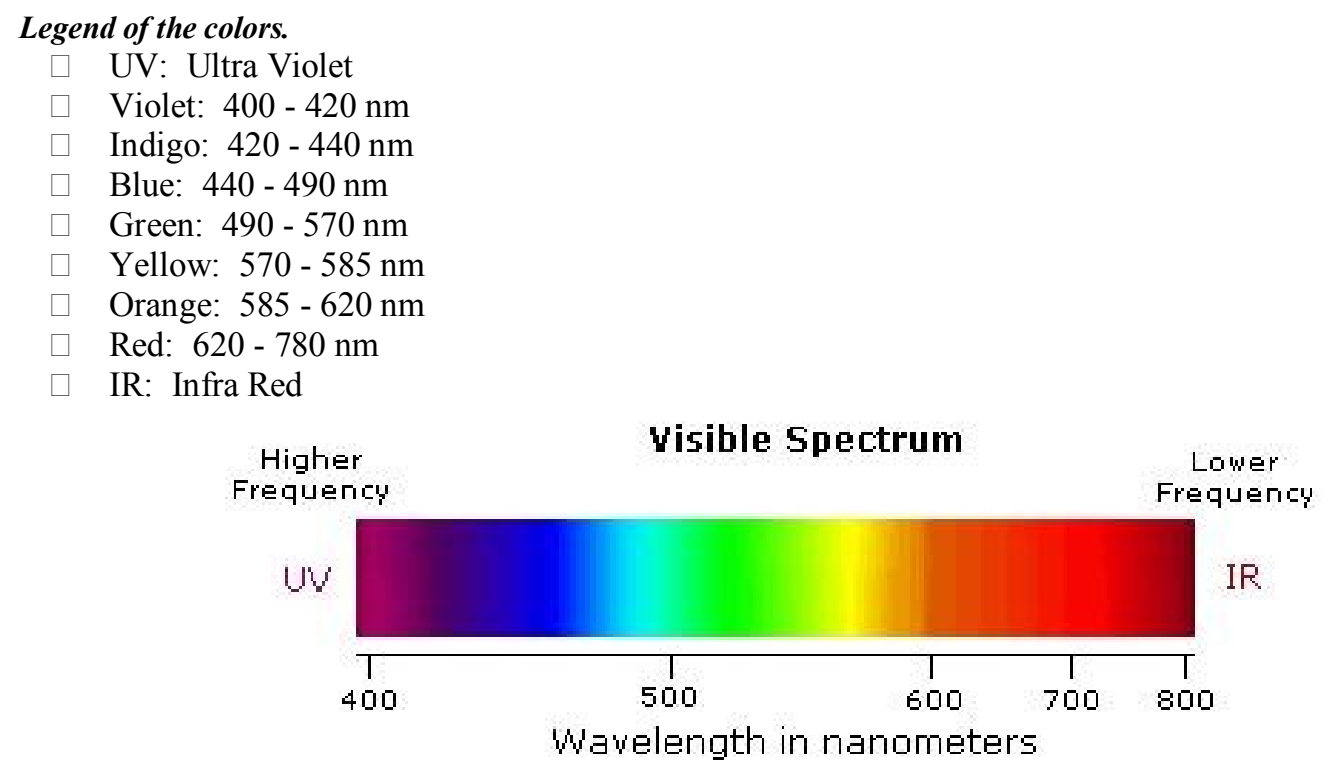

Figure 2. Electromagnetic spectrum

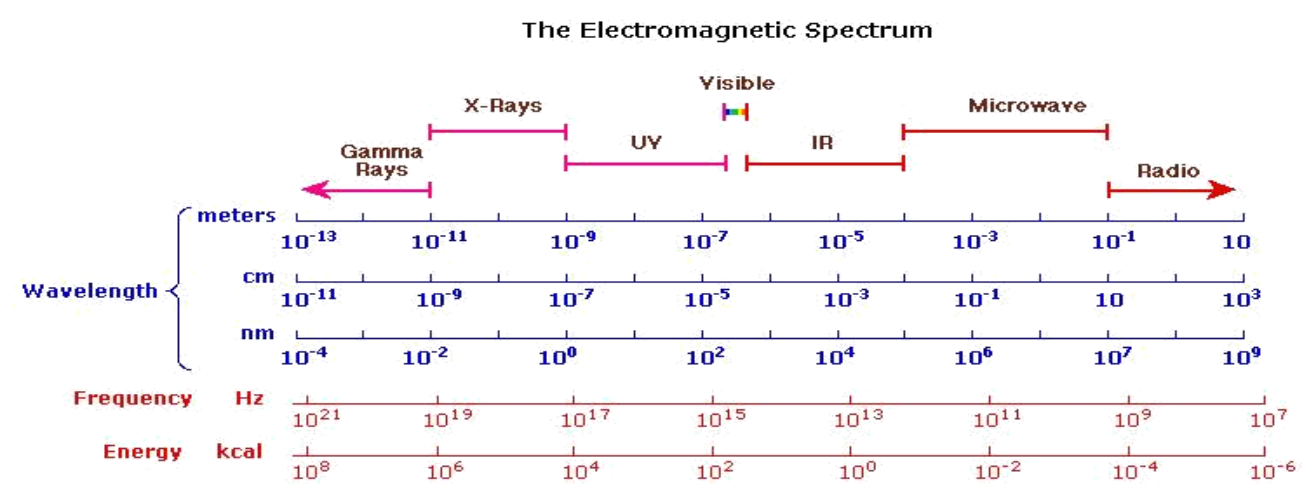

Figure 3. The full range of Electromagnetic spectrum

\section{Present Techniques}

Satellite instruments monitoring stratospheric ozone measure the decrease in intensity in UV solar radiation due to ozone absorption. The total Ozone mapping spectrometer on the Earth probe satellite 
(TOMS/EP) scans back and forth beneath the satellite to detect six individual frequencies of UV light that are scattered by air molecules back through the stratosphere. The more Ozone in the stratosphere, the more "backscattered" UV radiation will be absorbed compared to UV radiation directly from the Sun. The greenhouse gases $\mathrm{CO}_{2}$, nitrous oxide, and methane may also be monitored by IR spectroscopy.

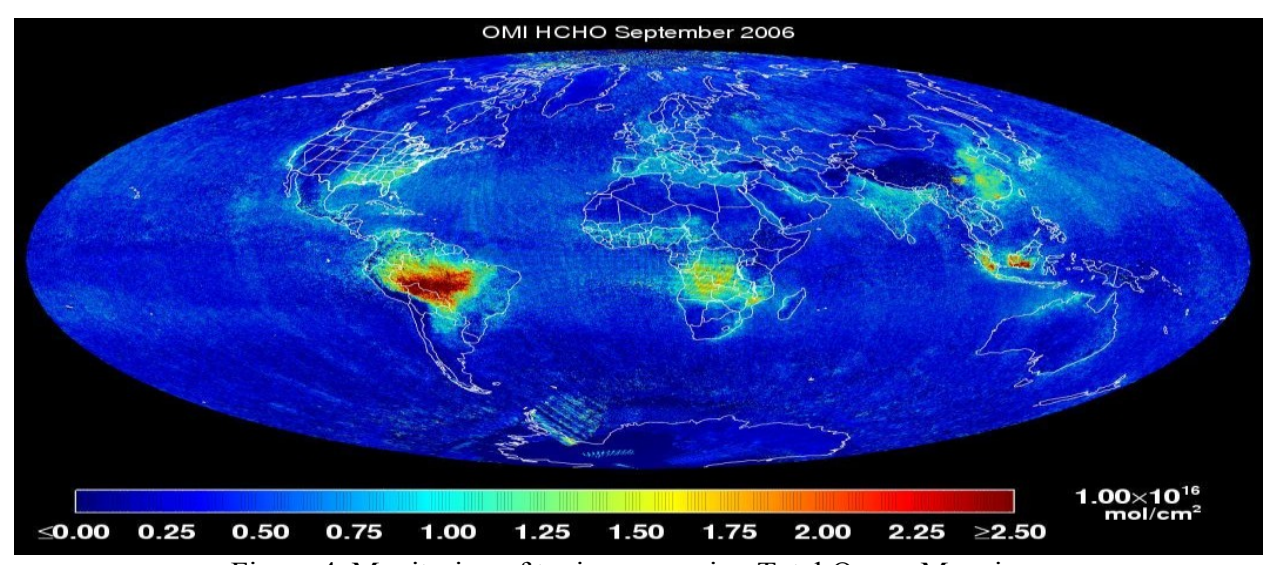

Figure 4. Monitoring of toxic gases using Total Ozone Mapping

\section{Description Of The Apparatus}

This safety apparatus monitoring the gases encompasses a field of view (FOV), comprising of a focusing element for focusing an infra-red (IR) energy beam collected from within the volume of space; a filter element for filtering the infra-red (IR) energy collected from within the volume of space; a micro electromechanical system (MEMS) having mirror elements in a mirror array for reflecting the IR energy beam; an IR energy detector for detecting the IR energy reflected by the MEMS array and converting the IR energy to an output signal. It also consists of an amplifier for amplifying the output signal; an analog to digital converter for converting the output signal from analog to digital; a processor for processing the output signal; a memory storage for storing the output signal; a controller for adjusting an angle of at least one mirror element of said MEMS mirror array; and an alarm for annunciating detection of an intrusion resulting from a change in amplitude of the output signal corresponding to a change in amplitude of the IR energy beam.

\section{Calibration Using Fourier Transform IR Spectroscopy (FTIR)}

Analyzes the absorption spectrum of a gas mixture to detect as many as twenty gases simultaneously. The technique involves analyzing the spectra mathematically and then comparing the observed fingerprints with calibrated reference spectra stored on the hard drive of the computer to be used for analysis.

Reference spectra for more than one hundred compounds are stored.

Instruments that use UV Fourier transform analysis are now available, which can be utilized for distinguishing between different gases and generating the accurate concentration levels. The hardware system will be fed with all the data such as wavelength, atomic number, etc. It applies mathematical Fourier transform method for comparing and observing the value obtained from the spectral fingerprint.

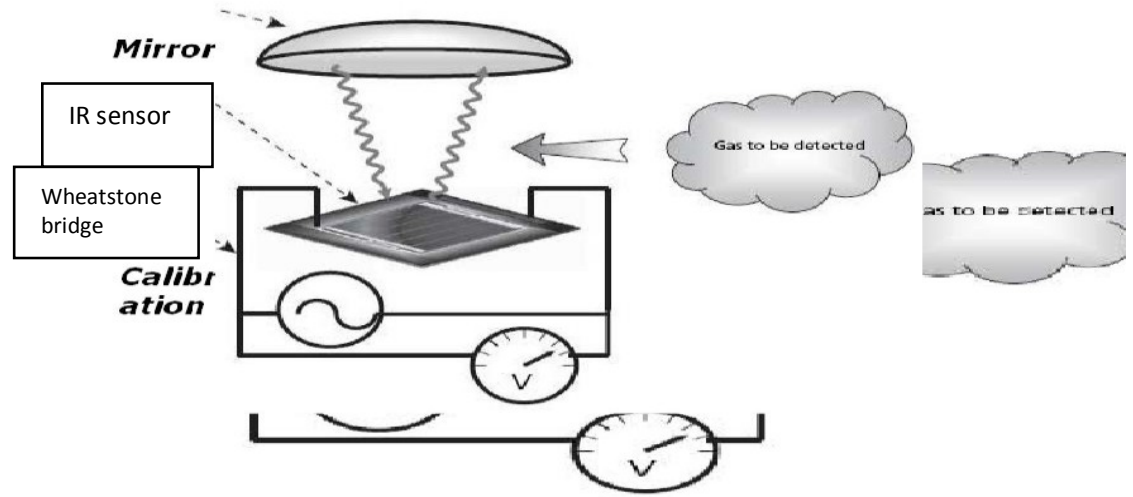

Figure 5. Sensing Mechanism 


\section{Conclusion}

Thus the system can be an effective scheme for the monitoring and detection of harmful gases which are posing a huge threat to the life on Earth. For example, Ammonia is often used as a coolant for turbine generators in power plants. It can be monitored for worker safety by its IR spectrum. Hydrogen fluoride, an extremely toxic gas used in the aluminum smelting and petroleum industries can be monitored to one part per million (ppm).The greenhouse gases carbon dioxide, nitrous oxide, and methane may also be monitored by IR spectroscopy.

\section{References}

[1] F. Kaczmaarck, "Infrared to Visible upconversion” Quantum electronics' lab, Institute of Physics, A. Mickiewicz University, Poznan.

[2] MEMS based safety infrared sensor apparatus and method. United States Patent 7145455

[3] P. K. Bhartia NASA Goddard Space Flight Center, Greenbelt, Maryland, USA, E. J. Welton NASA Goddard Space Flight Center, Greenbelt, Maryland, USA, Journal of Geophysical Research, Vol. 110,D10S18, doi:10.1029/2004JD004611, 2005

[4] Levelt, et al. Geoscience and Remote Sensing, IEEE Transactions on, Vol.44, Iss.5, May 2006 pp. 1199-1208

[5] www. pollutionissues.com 